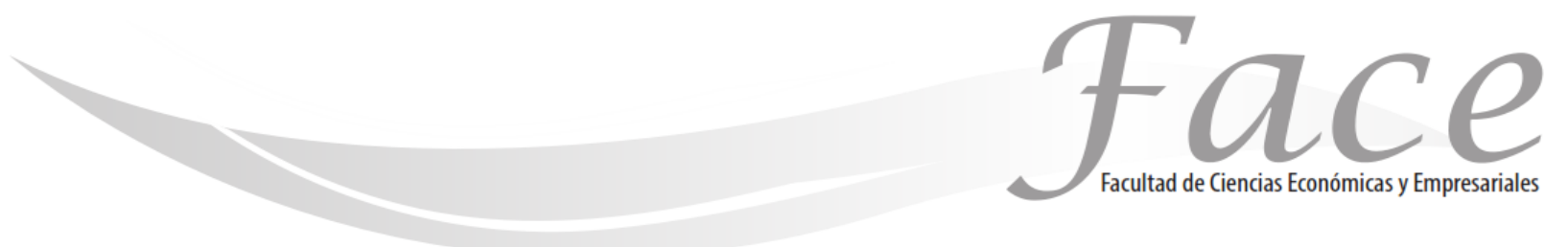

ISSN Impreso: 1794-9920

ISSN Electrónico: 2500-9338

Volumen $19-\mathrm{N}^{\circ} 1$

Año 2019

Págs. $58-69$

\title{
LOS MILLENNIALS DE TULANCINGO, HIDALGO Y EL COMERCIO ELECTRÓNICO
}

(c) (i) $(9)$

\author{
Irma Cárdenas García * \\ ORCID: http://orcid.org/0000-0003-3852-4101 \\ Santa Adali Vázquez Pimentel * * \\ ORCID: http://orcid.org/0000-0002-2241-2028 \\ Leonel Javier Hernández García \\ ORCID: http://orcid.org/0000-0002-5834-6771
}

Fecha de Recepción: Febrero 19 de 2019

Fecha de Aprobación: Mayo 10 de 2019

\section{Resumen:}

La presente investigación pretende conocer el nivel de uso y preferencias que tiene la Generación Millennials del Municipio de Tulancingo, Hidalgo, en cuanto al Comercio Electrónico, para ser considerado como estrategia de ventas, específicamente en tres vertientes: datos de tipo sociodemográfico, factores relativos al uso de dispositivos móviles y factores relativos al comercio electrónico. Siendo esta una investigación mixta, analizando una muestra formada por 382 cuestionarios aplicados a la generación Millennials nativa de este municipio que según los años de nacimiento se encuentran entre los 18 y 38 años de edad, demostrando así que el uso de la tecnología entre los pertenecientes a dicha generación está incorporada a su vida cotidiana principalmente en la adquisición y uso de los teléfonos inteligentes o Smartphones indicados como los dispositivos móviles de su predilección, mostrando una actitud positiva hacia el comercio electrónico para adquirir productos y/o servicios, pudiendo ser considerado como estrategia de ventas, pero siempre y cuando se realice un análisis de la forma de pago, ya que a pesar de que gran parte de los encuestados percibe como seguras las adquisiciones en línea, se deberá pensar en la estrategia a aplicar en cuanto a forma de pago, que brinde seguridad y confianza a ésta generación.

Palabras clave: Millennials, Dispositivos móviles, Comercio electrónico.

* Profesor Investigador Área de Desarrollo de Negocios, Universidad Tecnológica de Tulancingo, México; Magister en Administración de Negocios Área Mercadotecnia, Universidad TecMilenio, México Contacto: irmacg@utectulancingo.edu.mx

*** Profesor Investigador Área de Desarrollo de Negocios, Universidad Tecnológica de Tulancingo, México; Maestría en Dirección Comercial, Universidad Politécnica Tulancingo, México. Contacto: adali.vazquez@utectulancingo.edu.mx

*** Profesor Investigador Área de Contaduría, Universidad Tecnológica de Tulancingo, México. Maestría en Contribuciones Fiscales, Universidad Politécnica de Tulancingo, México. Contacto: leonel.hernandez@utectulancingo.edu.mx 


\title{
THE MILLENNIALS OF TULANCINGO, HIDALGO AND E-COMMERCE
}

\begin{abstract}
:
This research seeks to know the level of use and preferences of the Millennial Generation of the Municipality of Tulancingo, Hidalgo, in terms of E-Commerce, to be considered as a sales strategy, specifically in three areas: sociodemographic data, factors related to the use of mobile devices and factors related to e-commerce. This being a mixed investigation, analyzing a sample formed by 382 questionnaires applied to the native Millennials generation of this municipality that according to the years of birth are between 18 and 38 years of age, demonstrating that the use of technology among those belonging to this generation is incorporated into their daily lives mainly in the acquisition and use of Smartphones or Smartphones indicated as the mobile devices of their choice, showing a positive attitude towards electronic commerce to acquire products and / or services, which can be considered as sales strategy, but only when an analysis of the payment method is carried out, since despite the fact that a large part of the respondents perceive online acquisitions as safe, the strategy to be applied in terms of the form of payment, which provides security and confidence to this generation.
\end{abstract}

Keywords: Millennials, Mobile devices, E- commerce.

\section{OS MILÊNIOS DE TULANCINGO, HIDALGO E COMÉRCIO ELETRÔNICO}

Resumo:

Esta pesquisa busca conhecer o nível de uso e preferências da Geração Milenar do Município de Tulancingo, Hidalgo, em termos de Comércio Eletrônico, para ser considerada como uma estratégia de vendas, especificamente em três áreas: dados sociodemográficos, fatores relacionados ao uso de dispositivos móveis e fatores relacionados ao comércio eletrônico. Sendo esta uma investigação mista, analisando uma amostra formada por 382 questionários aplicados à geração Millennials nativa do município This que de acordo com os anos de nascimento estão entre 18 e 38 anos de idade, demonstrando que o uso da tecnologia Entre os que pertencem a esta geração é incorporado em suas vidas diárias, principalmente na aquisição e uso de smartphones ou Smartphones Indicado como os dispositivos móveis de sua escolha, mostrando uma atitude positiva para 0 comércio eletrônico para adquirir produtos e / ou serviços, que pode ser considerado como estratégia de vendas, mas apenas quando uma análise do método de pagamento é realizado, uma vez que apesar do fato de que uma grande parte dos entrevistados percebem aquisições online como seguro, a estratégia a ser aplicada em termos da forma de pagamento, o que proporciona segurança e confiança para esta geração.

Palavras-chave: Millennials, dispositivos móveis, comércio eletrônico. 


\section{INTRODUCCIÓN:}

Martínez (2011) define al dispositivo móvil como "un aparato de pequeño tamaño, con algunas capacidades de procesamiento, alimentación autónoma, conexión permanente 0 intermitente a una red, memoria limitada, diseñado específicamente para una función, pero que puede llevar a cabo otras funciones más generales", entre los dispositivos móviles se tienen los smartphones, tabletas, reproductores de música, computadoras portátiles y consolas de videojuegos; en la actualidad muchas personas acceden a la red mediante estos dispositivos, pues se pueden hacer infinidad de cosas como: leer libros, investigar, ver noticias, comprar, hacer transacciones bancarias, estudiar cursos, acceder a redes sociales, vender, comunicarse, etc. actividades en las que no se pueden dejar de lado las conocidas aplicaciones móviles ya que sin ellas sería muy complicado navegar en la red, surgiendo un gran cuestionamiento ¿Quiénes usan estas herramientas para hacer más prácticas las actividades?

Resultado imperante que la "Generación Millennials" ha adoptado las tecnologías para el desarrollo de sus múltiples actividades, perteneciendo a esta generación los sectores de la población nacidos entre 1980 y el 2000, cuya edad oscila entre los 19 y 39 años de edad, también llamada generación " $Y$ " o generación Net, resultando afortunados, porque crecieron con los conocimientos necesarios para manipular cualquier dispositivo tecnológico, pero, en la mitad de los casos, también recuerdan cómo era el mundo antes del boom del internet (Merca 2.0, 2013).

Montoya Camacho \& Orozco Ramírez (2017) afirman que actualmente los Millennials forman parte de un $75 \%$ de los usuarios que navegan en internet a diario, resultando de suma importancia determinar el tipo de estrategias para este nicho, punto primordial para alcanzar el éxito en el mercado demandante en el que vivimos. Para esta generación es fundamental brindarles contenido de valor y concretar el proceso de vender, es por eso que rechazan lo obsoleto y dedican el mayor tiempo de su vida a interactuar con los dispositivos electrónicos ya sea revisando información, jugando o comprando. (Montoya Camacho \& Orozco Ramírez, 2017)

Todo esto detona la realización de la presente investigación para conocer el nivel de influencia que tiene el comercio electrónico en la Generación Millennials del Municipio de Tulancingo, estructurándola en cuatro apartados: el primero consta de los trabajos realizados con respecto a esta generación, el uso de los dispositivos móviles y el comercio electrónico; el segundo contiene el diseño y la metodología a utilizar en la investigación; en el tercero se analizan los resultados obtenidos; y por último en el apartado número cuatro se mencionan las conclusiones y las aportaciones de la investigación realizada.Objetivo general: La presente investigación pretende conocer el nivel de uso y preferencias que tiene la Generación Millennials del Municipio de Tulancingo, Hidalgo, en cuanto al Comercio Electrónico, para ser considerado como estrategia de ventas.

\section{MARCO TEÓRICO:}

\section{Los Millennials}

Según Caraher (2015) la "Generación Y" comúnmente conocida como "Millennial" es la generación que nació entre el año 1980 y el año 2000, así que los Millennials actualmente rondan entre los 39 y 19 años de edad proyectándose que para el año 2025 serán el $75 \%$ de la fuerza laboral del mundo (Gayá, 2017).

Penagos \& Rubio (2015) hacen hincapié en que aprender de los Millennials y entenderlos no es solo para estar informados acerca de sus perspectivas y su modus operandi, sino por un tema de progreso de culturasociedad de cara a un futuro próximo con líderes globales de alto desempeño. Asimismo, la tecnología y la comunicación inmediata es un hito que marca varias diferencias con las generaciones anteriores, siendo estos los primeros nativos digitales. Estos ya no conciben el trabajo como un lugar físico. La impaciencia es también fruto de la comunicación inmediata y el tiempo en el que avanza todo el mundo.

La convergencia digital se ha incorporado en su cotidianidad, esta generación ha roto el molde y los clichés; lo suyo es reinventar, inventar, innovar y emprender nuevos desafíos constantemente. (Álvarez, 2014)

Los millennials según Gayá (2017) son sociales, conectados, preocupados por su imagen, aunque siempre han sido los niños egocéntricos, son, según el estudio de MetLife, los que tienen una mayor predisposición a cuidar de su familia. Tienen muy en cuenta la importancia de ayudar a los niños, quizás por el soporte incesante de sus padres. Y lo que más les influencia en la toma de decisiones es la familia, seguida por el ámbito educativo y los amigos.

La revista Forbes les adjudica seis rasgos clave: son nativos digitales, sus relaciones básicas y cotidianas están intermediadas por una pantalla, o por varias, la que es su segunda característica; son multipantalla, de comportamiento multitasking, con capacidad o necesidad de hacer dos cosas a la vez, hablan otro lenguaje, la publicidad clásica o la televisión entendida como hasta 
ahora no es para ellos. Y es que los millennials son adictos al móvil, a las aplicaciones; casi la mitad confiesa que no podría pasar un día sin su móvil. Son muy sociales, y lo demuestran en las redes, son exigentes y críticos, valoran mucho que las empresas los consideren como clientes, tanto, que están modificando sus estrategias, dando más valor a la experiencia de compra que al propio producto. Los millennials exigen personalización y valoran la colaboración, están muy atentos a aspectos como la transparencia, la sostenibilidad y el compromiso social. Esta generación ha roto los patrones de consumo respecto a las anteriores generaciones. Buscan la información en Internet, sus procesos de compra son altamente sociales y comparten tanto sus compras como sus intenciones de compra. Los tradicionales canales de publicidad van desapareciendo y las empresas tienen que prestar mucha más atención a las redes sociales y a la atención al cliente (Gayá, 2017).

En el sitio actualidad.rt.com (RT, 2015) se publicó el artículo titulado: "Millennials: Así es la generación que ya no recuerda cómo era el mundo sin Internet" que muestra las características de los pertenecientes a dicha generación entre las que se encuentran:

1. Nacieron entre los años 80 y la pasada década, crecieron en una era de rápido desarrollo de las nuevas tecnologías, y casi no recuerdan cómo era el mundo sin Internet.

2. Son idealistas e impacientes y están bien preparados académicamente.

3. Son inmaduros, individuales, tienen altas expectativas, confianza en sí mismos, autoestima inflada y la necesidad de comodidad, además poseen capacidad de adaptarse a nuevas condiciones y cambiar de hábitat y de trabajo, así como la de crecer rápidamente y resolver varios problemas simultáneamente, quieren todo a la vez.

4. No soportan un trabajo poco interesante y rutinario, no les gusta dejar las cosas buenas para luego.

5. Quieren dejar su huella en la historia, vivir una vida interesante, formar parte de algo grande, crecer y desarrollarse, cambiar el mundo que les rodea, y no solo ganar dinero.

6. La generación del milenio es mucho menos religiosa que las generaciones precedentes.

7. Los científicos también señalan que el ritmo de vida de estas personas es mucho más rápido, por lo que a menudo se muestran muy impacientes. Asimismo, a diferencia de las generaciones precedentes, entre ellos hay muy pocas personas adictas al trabajo, ya que aspiran a mantener el equilibrio entre su carrera laboral y su vida personal.

8. El uso activo de las nuevas tecnologías diferencía a estos jóvenes de las otras generaciones, especialmente por la necesidad de compartir información.

Según Franichevich \& Marchiori (2008) los Millennials son una generación muy estudiada por los investigadores porque muchos los consideran como lo "nuevo", lo "diferente", lo "molesto" para las generaciones que les anteceden, debido a que no son fáciles de comprender ya que traen nuevos paradigmas, entre ellos la manera de usar la tecnología para comunicarse, divertirse, trabajar y comprar, esta última característica los pone en la mira de las empresas como un mercado potencial para hacer comercio electrónico, pues es bien sabido que la importancia que los Millennials dan a los dispositivos móviles es abrumadora, nunca se separan de ellos tanto así que es lo primero que miran al levantarse.

\section{El papel de la tecnología}

Los Millennials tienen como característica principal el uso permanente de la tecnología prueba de ello es la Encuesta Nacional Sobre Disponibilidad y Uso de Tecnologías de la Información en los Hogares (2016), donde queda cada vez más claro que aumenta el uso de internet, teléfonos inteligentes y tv digital en México, los resultados que arrojó esta encuesta son que el número de usuarios de teléfonos inteligentes o smartphones creció de 50.6 millones a 60.6 millones entre 2015 y 2016, en contraste, el total de usuarios de computadora disminuyó 4.3 puntos porcentuales en el mismo periodo. Las principales actividades de los internautas mexicanos son comunicarse $(88.9 \%)$, tener acceso a contenidos audiovisuales $(81.9 \%)$ y entretenimiento $(80.1 \%)$. En cuanto a conectividad, esta encuesta reveló que 15.7 millones de hogares disponen de conexión a Internet (47.0 por ciento del total nacional) ya sea mediante una conexión fija o de una de tipo móvil, lo que significa un incremento de 7.8 puntos porcentuales respecto a la penetración del año anterior y un incremento 41 puntos con respecto al año 2001 demostrándose una tendencia al alza anualmente (INEGI, 2016). (Ver imagen 1.1) 
Imagen $N^{\circ} 1.1$

Hogares con internet, 2001-2006.

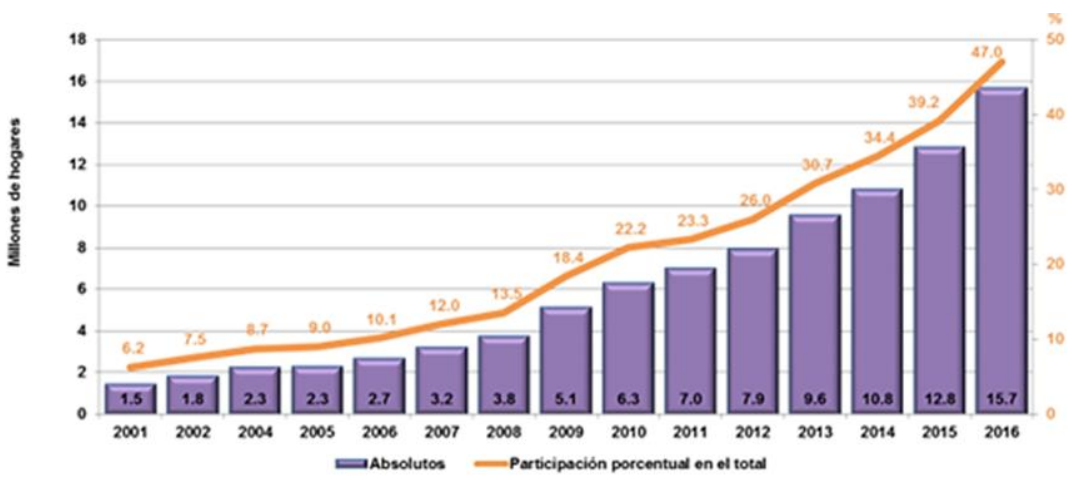

Fuente: INEGI. (2016). Encuesta Nacional Sobre Disponibilidad y Uso de Tecnologías de la Información en los Hogares, 2016. Aguascalientes, Ags.: INEGI.

Algo muy importante es que 65.5 millones de personas de seis años 0 más utilizan Internet presentando un crecimiento de 2.1 puntos porcentuales más que en año 2015. (Ver imagen 1.2)

Imagen 1.2:

Población según condición de uso de Internet, 2016.

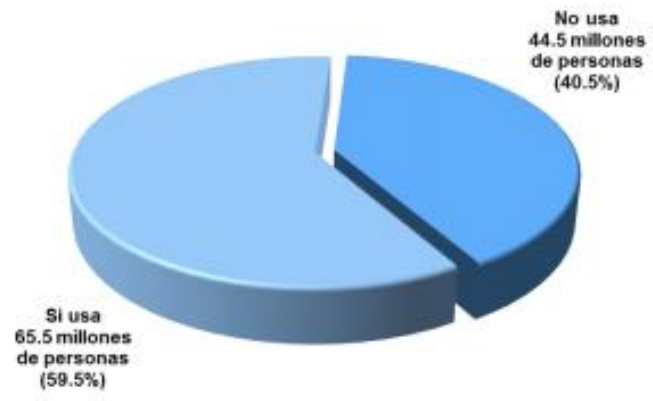

Fuente: INEGI. (2016). Encuesta Nacional Sobre Disponibilidad y Uso de Tecnologías de la Información en los Hogares, 2016. Aguascalientes, Ags.: INEGI.

La encuesta también muestra que de acuerdo a las edades los jóvenes de entre los 18 a 24 años y 25 a 34 años son los que hacen más uso del Internet con el 85 y 74.3 por ciento respectivamente, es importante mencionar que entre estos rangos entran los considerados como Millennials. (Ver imagen 1.2)

\section{Imagen 1.2:}

Población por grupos de edad según condición de uso de Internet, 2016.

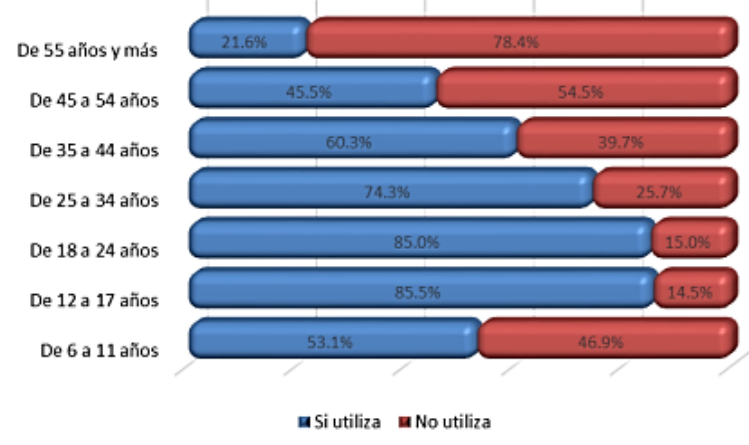

Fuente: INEGI. (2016). Encuesta Nacional Sobre Disponibilidad y Uso de Tecnologías de la Información en los Hogares, 2016. Aguascalientes, Ags.: INEGI.

De este mismo rango de edad según el género las que más usan Internet son las mujeres con el $51.2 \%$, mientras el $48.8 \%$ son hombres. (Ver imagen 1.3 ) 
Imagen 1.3:

Usuarios de internet por género y edad.

\begin{tabular}{|l|c|c|}
\hline Usuarios de Internet por género y edad & Mujeres & Hombres \\
\hline Adultos mayores: de 60 años o más & $49.5 \%$ & $\mathbf{5 0 . 5} \%$ \\
\hline Adultos: de 35 a 59 años & $53.2 \%$ & $46.8 \%$ \\
\hline Jóvenes: de 18 a 34 años & $51.2 \%$ & $48.8 \%$ \\
\hline Menores: de 6 a 17 años & $50.6 \%$ & $49.4 \%$ \\
\hline
\end{tabular}

Fuente: INEGI. (2016). Encuesta Nacional Sobre Disponibilidad y Uso de Tecnologías de la Información en los Hogares, 2016. Aguascalientes, Ags.: INEGI.

Por su parte la Encuesta Global de Nielsen (NIELSEN, 2015) menciona que la tecnología está transformando radicalmente la forma en la que los usuarios se relacionan con el mundo, incluyendo la forma, trabajar y comunicarse, obteniendo que el $82 \%$ de los mexicanos ve películas en TV mientras que el $12 \%$ lo hace en su smartphone, el $14 \%$ tiene citas electrónicas, como pasatiempo el $53 \%$ usa el smartphone. En cuanto al propósito de los mexicanos para estar conectados según la encuesta el $85 \%$ lo hace para estar en contacto con la familia y amigos, el $55 \%$ para buscar trabajo y un $15 \%$ para comprar alimentos. (Ver imagen 1.3)

\section{Imagen 1.4:}

Propósito de los mexicanos para estar conectados a internet.

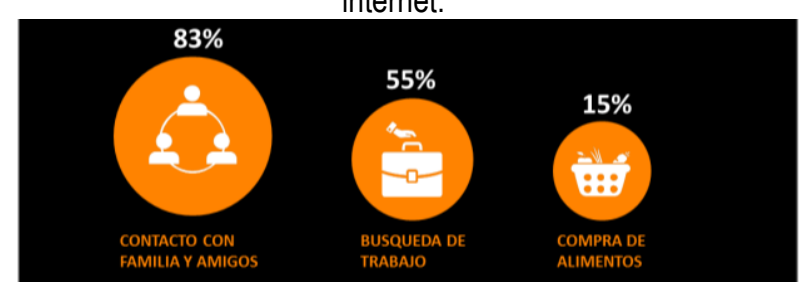

Fuente: NIELSEN. (2015). Encuesta global Nielsen al tercer trimestre de 2014: Panorama digital mundial. [EUA]: Nielsen.

Los Millennials pasan gran parte de su tiempo conectados a internet, es ahí donde interactúan y también donde compran, usando para ello los denominados smartphones de acuerdo con los resultados de la ENDUTIH 2016, tres de cada cuatro usuarios de celular disponen de uno y el 81 por ciento dispone de conexión móvil, lo que les permite el acceso a Internet desde cualquier lugar. (Ver imagen 1.5)
Imagen 1.5: Usuarios de telefonía celular según equipo, 2016.

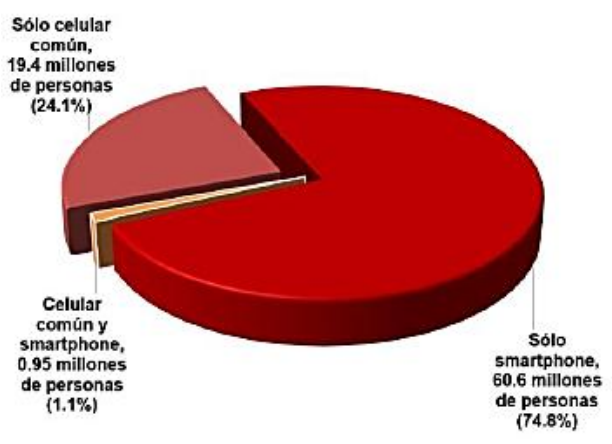

Fuente: INEGI. (2016). Encuesta Nacional Sobre Disponibilidad y Uso de Tecnologías de la Información en los Hogares, 2016. Aguascalientes, Ags.: INEGI.

\section{Comercio electrónico}

Según los estudios de la Organización para la Cooperación y el Desarrollo Económicos (OCDE) el comercio electrónico se define como el proceso de compra, venta o intercambio de bienes, servicios e información a través de las redes de comunicación. Representa una gran variedad de posibilidades para adquirir bienes 0 servicios ofrecidos por proveedores en diversas partes del mundo (PROFECO, [2018]).

En 2016 la AMIPCI realizó el Estudio de Comercio Electrónico en México 2016, encuestando a un total de 1829 personas con edades de que oscilan de los 18 a los 55 años teniendo como edad media 36 años, contactadas vía correo electrónico, los objetivos de estudio fueron: cuantificar el valor y volumen del comercio electrónico en México por tipo de dispositivo y categoría, comprender las preferencias y el nivel de satisfacción de los compradores digitales, derivado de ese estudio se obtienen los siguientes datos relevantes el primero es que la penetración de dispositivos fue alta con casi la mitad de los compradores en línea utilizando principalmente tres dispositivos: Computadora, Smartphone y Tablet; siendo los compradores de 18 a 34 años de edad los que presentaron una mayor posibilidad de tener que usar un Smartphone en comparación con otras edades. Pero en general el $48 \%$ de los encuestados utilizan los tres dispositivos (Computadora, Smartphone y Tablet), el 33\% utilizan computadora y Smartphone, el $8 \%$ solo computadora, el $6 \%$ sólo Smartphone, el 1\% computadora y Tablet y el $4 \%$ solo Smartphone y Tablet. (Ver Imagen 1.6). 
ISSN: 1794-9920 Impreso / Electrónico 2500-9338

Enero - Julio de 2019 - Volumen 19 Número 2, Año 2019 Págs. 58 - 69

Imagen 1.6:

Penetración de dispositivo.

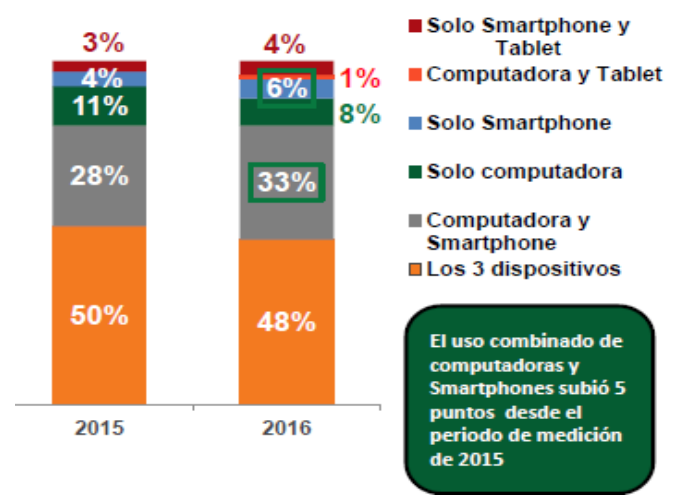

Fuente: AMIPCI, 2016.

El mismo estudio menciona que los tres tops categorías de compras en línea fueron: ropa y accesorios, descargas digitales y boletos para eventos con el 59,48 y $36 \%$ respectivamente. En comparación con los compradores de computadoras, los consumidores que utilizan dispositivo móvil señalan que el uso de aplicaciones les ahorra tiempo y la capacidad de acceder a Internet desde cualquier lugar $y$ en cualquier momento son motivos para utilizar sus dispositivos para la compra en línea. Cabe mencionar que tres de cada cinco usuarios de dispositivo móvil comentaron que habían utilizado sus dispositivos para investigar, comparar precios, los detalles del producto, y encontrar las tiendas cercanas. Los compradores en línea mencionan que entre los tipos de pagos más usados son el Paypal con $70 \%$, seguido de la Tarjeta de debido con el $61 \%$ y en tercer lugar se ubica la Tarjeta de crédito personal con el $53 \%$.

Miller (2017), menciona en su artículo para Milenio.com denominado: "Paradojas de los Millennials a la mexicana" que un rasgo notable de la generación Millennials es que se considera la primera nativa en la era digital, lo que la hace proclive a todo tipo de consumo por esta vía: moda, música, videos, libros, etc., siendo una generación que genera vínculos afectivos, de amistad, políticos y culturales empleando un escaparate virtual. Una cifra aproximada de Millennials en México está cercana a los 46 millones (según el Censo de Población y Vivienda 2010) y la autora asegura que han vivido en la inmediatez, debido a que no saben esperar pues Internet les provee prácticamente de todo casi de forma instantánea.

La generación $Y$ o Millennial según Rogelio Simonato \& Ariel Mori (2015) nace en un mundo en que el consumismo esta socialmente aceptado y profundamente arraigado como estilo de vida. A través de este mayor consumo
Bauman (2010) observa la constante actualización del uso de la tecnología, la misma que está disponible para estar conectado a Internet, y que les da mayor libertad de la que tuvieron los jóvenes de los 60 en nuestro país, o aquellos que participaron en las movilizaciones del mayo francés en el 68.

Muchos investigadores han observado que el comercio electrónico representa un cambio importante en la manera en que se llevan a cabo los negocios y lo comparan con otros cambios históricos en la organización económica, como la Revolución Industrial. Desde el año 2001 los analistas de la industria han predicho el surgimiento del comercio basado en la telefonía móvil (a menudo llamado comercio móvil o m-comercio). El comercio móvil incrementa gracias al uso cada vez más generalizado de los teléfonos celulares que permiten el acceso a la internet y de los teléfonos inteligentes; éstos son celulares que incluyen un explorador web, un teclado completo y un sistema operativo identificable que permite a los usuarios ejecutar diversos paquetes de software, y se encuentran disponibles con paquetes de planes de transferencias de datos 0 , incluso, ilimitados a cambio de una cuota mensual fija. Uno de los cambios más importantes que se generó a raíz de la operatividad total de los dispositivos portátiles es que la Internet en realidad está disponible en cualquier lugar; esto puede cambiar la conducta de los compradores en diversas formas y estas áreas de oportunidad sirven para que las empresas tomen en cuenta a estos usuarios de la tecnología y le den mayor importancia al comercio digital.

\section{METODOLOGIA:}

\section{Descripción de la muestra}

Hernández, Fernández \& Baptista (2010), comentan que uno de los puntos cruciales de cualquier investigación es la de determinar a los sujetos que serán investigados para alcanzar el objetivo propuesto, habiendo decidido que por el tamaño de la población se determinaría una muestra, aplicando la fórmula de población finita, pues según datos del Censo de Población y Vivienda (2010), la población total del Municipio de Tulancingo Hidalgo es de 151,584 habitantes de los cuales 53,727 (35.4\%) pertenece a la generación Millennials, los cuales oscilan entre los 18 y 38 años de edad. Determinando la siguiente muestra:

$$
n=\frac{z^{\wedge} 2\left(p^{\star} q\right)}{e^{\wedge} 2+\frac{\left(z^{\wedge} 2\left(p^{\star} q\right)\right)}{N}}
$$

$\mathrm{n}=$ Tamaño de la muestra

$z=$ Nivel de confianza deseado

$\mathrm{p}=$ Proporcion de la poblacion con la caracteristica deseada (exito)

$\mathrm{q}=$ Proporcion de la poblacion sin la caracteristica deseada (fracaso)

$\mathrm{e}=$ Nivel de error dispuesto a cometer

$\mathrm{N}=$ Tamaño de la poblacion 
Tabla 1: Muestra

\begin{tabular}{cc}
\hline & TULANCINGO \\
\hline Hombres & 165 \\
Mujeres & 217 \\
Totales & 382 \\
\hline Fuente: Elaboración propia (2018)
\end{tabular}

\section{Alcance de la investigación}

La investigación a realizar es mixta por un lado cualitativa de tipo etnográfico ya que se estudió por medio de la teoría solo a la generación Millennials y sus características, y por otro lado es cuantitativa descriptiva no experimental pronosticando que la generación Millennials de Tulancingo Hidalgo está ligada al uso de los dispositivos móviles para adquirir productos 0 servicios mediante el comercio electrónico.

\section{Diseño de la encuesta}

Para recabar información se diseñó un cuestionario que fue útil para conocer el nivel de uso y preferencias que tiene la Generación Millennials del Municipio de Tulancingo, Hidalgo, en cuanto al Comercio Electrónico. La encuesta está conformada por 15 ítems, cada una con un objetivo diferente para recopilar información, las preguntas fueron estructuradas para conocer el consumo que tiene la generación estudiada en cuanto al comercio electrónico, el consumo de internet en los dispositivos móviles de su preferencia, plataformas más usadas para comprar en línea y forma de pago en las compras.

\section{Técnicas e instrumentos}

Se definió el segmento de estudio a hombres y mujeres de 18 a 38 años de edad residentes de la ciudad de Tulancingo Hgo. Se utilizó la fórmula finita que es el compuesto por una cantidad limitada de elementos, para el cálculo de tamaño de muestra cuando el universo es finito, es decir contable y la variable de tipo categórica, primero debe conocer "n" número total de habitantes del municipio, de esta manera tomar una muestra representativa para realizar las encuestas necesarias para que los resultados sean confiables.

El instrumento cumple con las pruebas de confiabilidad, validez y objetividad, utilizando el programa estadístico SPSS (Statistical Package for the Social Sciences) obteniendo un Alfa de Cronbach de .843, superior al $75 \%$, lo que indica que el instrumento es altamente confiable.
La estructura del instrumento consta de tres factores:

- Datos de tipo sociodemográficos

- Factores concernientes a los dispositivos móviles

- Factores relativos al comercio electrónico

\section{RESULTADOS:}

De acuerdo a la literatura previa y a través del análisis descriptivo, los resultados del comportamiento del índice de consumo de productos y/o servicios a través de comercio electrónico respecto de los datos de tipo sociodemográficos, factores concernientes a los dispositivos móviles y factores relativos a la compra en línea son presentados para estudiar el nivel de consumo de comercio electrónico por la Generación Millennial en el Municipio de Tulancingo (Hernández, Fernández \& Baptista, 2010). Son los siguientes.

De los 382 Millennials encuestados más de la mitad son mujeres con un $57 \%$, mientras que el porcentaje de hombres es de $43 \%$, (Ver gráfica 1).

Gráfica 1: Millennials de Tulancingo según género.

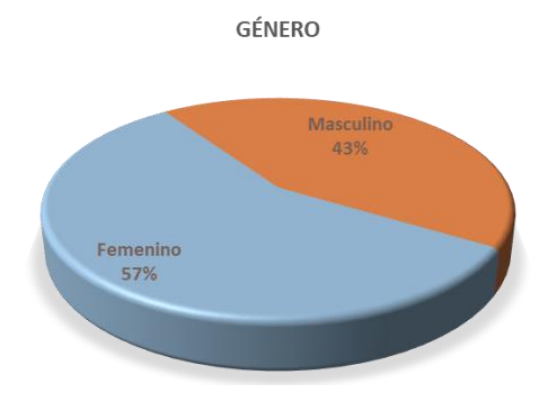

Fuente: Elaboración propia.

El rango de edad de los encuestados más representativo es el de 18 a 25 años ya que representa un $74 \%$, mientras que los encuestados de 26 a 33 años son un $23 \%$ y los restantes su edad oscila entre los 34 y 38 años, (Ver gráfica 2).

Gráfica 2: Distribución porcentual según rango de edad. 
ISSN: 1794-9920 Impreso / Electrónico 2500-9338

Enero - Julio de 2019 - Volumen 19 Número 2, Año 2019 Págs. 58 - 69

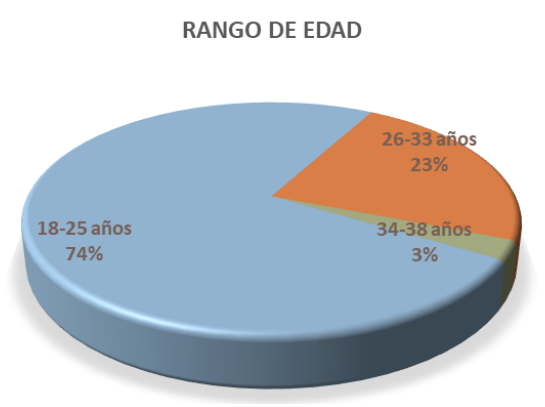

Fuente: Elaboración propia.

Casi la totalidad de los encuestados utiliza un dispositivo móvil (99\%) sólo una mínima parte de correspondiente al $1 \%$ no lo utiliza, (Ver gráfica 3).

Gráfica 3: Índice de consumo de dispositivos móviles. ¿UTILIZA ALGÚN DISPOSITIVO MÓVIL?

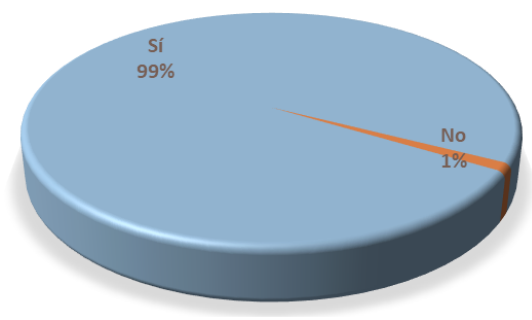

Fuente: Elaboración propia.

De los dispositivos móviles: Smartphone, Lap top y Tabletas, el índice de consumo es de $78 \%, 14 \%$ y $8 \%$ respectivamente, indicándonos que el Smartphone es el dispositivo electrónico de mayor consumo, (Ver gráfica 4).

Gráfica 4: Índice de consumo del dispositivo móvil. ¿QUÉ DISPOSITIVO UTILIZA CON MAYOR FRECUENCIA?

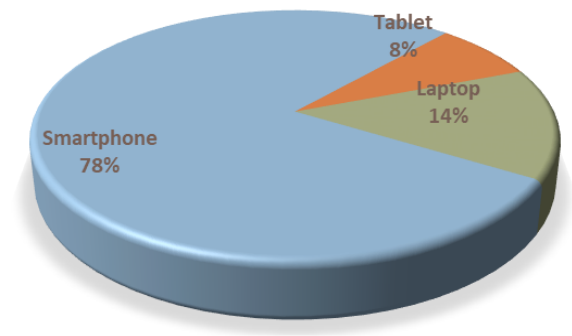

Fuente: Elaboración propia.

De los Millennials que utilizan un dispositivo móvil el 94\% menciona que tiene acceso internet la mayor parte del tiempo mientras que sólo el $6 \%$ no tiene acceso a internet, (Ver gráfica 5).

Gráfica 5: Índice de consumo Internet según uso de dispositivo móvil.

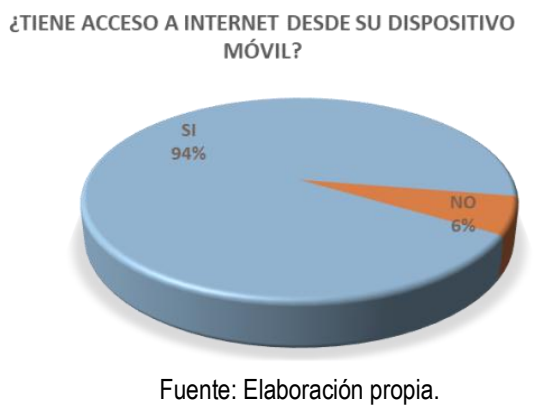

Es el hogar, el lugar en el que la mayoría de los encuestados (56\%) acceden a internet, mientras que el $24 \%$ su lugar de acceso es en escuelas, un $17 \%$ lo hace en lugares públicos y una mínima parte del $3 \%$ en la oficina, (Ver gráfica 6).

Gráfica 6: Índice de consumo Internet según lugar de acceso.

¿EN QUÉ LUGAR SUELE ACCEDER A INTERNET?

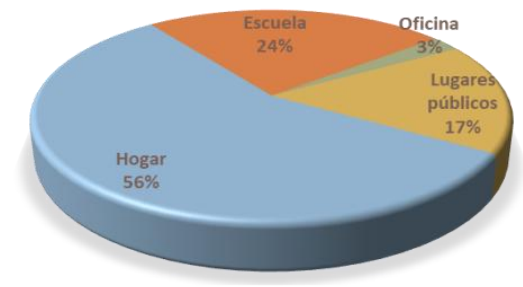

Fuente: Elaboración propia.

Aunque los Millennials son una generación que usa en gran manera el servicio de internet, se puede ver que no invierten mensualmente tanto en ello, el $45 \%$ invierte de 50 a 100 pesos, en segundo lugar, se encuentra de 101 a 200 pesos con el $37 \%$, en tercer lugar, se encuentran los que invierten más de 301 pesos con el $14 \%$ y en último lugar se encuentran los que inviernes de 201 a 300 pesos con un valor porcentual de $4 \%$, (Ver gráfica 7 ).

Gráfica 7: Índice de consumo Internet según inversión en pesos (mensualmente). 
¿CUÁNTO GASTA MENSUALMENTE EN EL USO DE SU DISPOSITIVO MÓVIL?

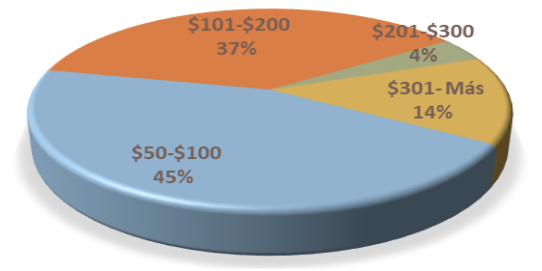

Fuente: Elaboración propia.

En relación a la importancia que los Millennials les dan a las aplicaciones, casi la mitad de los encuestados (47\%) consideran a las redes sociales como la aplicación más importante, mientras que un $26 \%$ se inclina por la mensajería instantánea y el $12 \%, 9 \%$ y $6 \%$ por la música, los juegos, y los editores de fotografías respectivamente, (Ver gráfica 8).

Gráfica 8: Índice de consumo de aplicaciones.

¿QUÉ APLICACIÓN CONSIDERA MÁS IMPORTANTE CUANDO USA SU DISPOSITIVO MÓVIL?

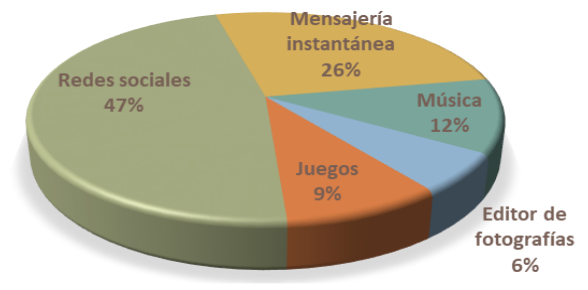

Fuente: Elaboración propia.

Con respecto a si los Millennials han pagado por descargar alguna App, el $53 \%$ menciona que sí, mientras que el $47 \%$ no lo ha hecho, (Ver gráfica 9 ).

Gráfica 9: Índice de consumo de aplicaciones según forma de obtención.

¿HA PAGADO POR DESCARGAR UNA APP?

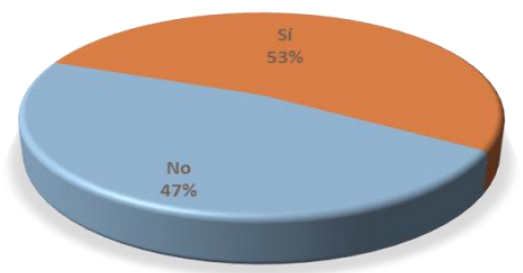

Fuente: Elaboración propia.

Los Millennials son personas que se caracterizan por querer obtener productos y servicios con rapidez, en esta gráfica se muestra que el $57 \%$ ha comprado alguna vez en línea mientras que el $43 \%$ nunca lo ha hecho, (Ver gráfica 10).

Gráfica 10: Índice de compras en línea.

¿HA REALIZADO COMPRAS EN LÍNEA?

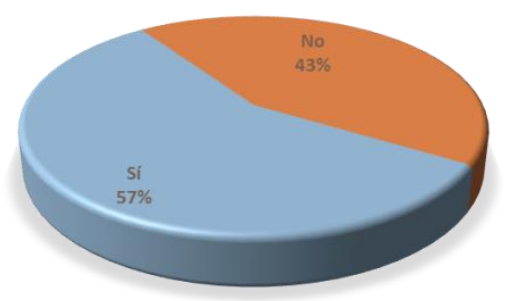

Fuente: Elaboración propia.

Del $57 \%$ de los Millennials que han comprado alguna vez por lo menos en Internet, la plataforma que más han utilizado son las Redes Sociales con el $50 \%$, seguido de Mercado Libre con el $26 \%$, en tercer lugar, está Amazon con el $15 \%$ y por último se encuentra la opción Otro con el $9 \%$. (Ver gráfica 11)

Gráfica 11: Índice de consumo de plataformas para compras en línea.

\section{¿QUÉ PLATAFORMA HA USADO PARA COMPRAR EN LÍNEA?}

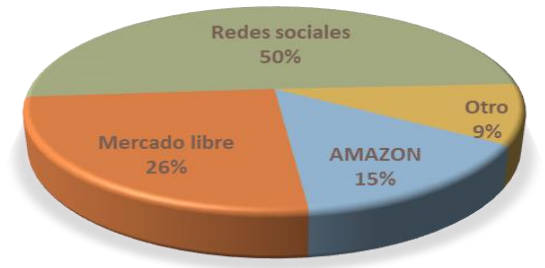

Fuente: Elaboración propia.

En cuanto a la forma de pago que utilizan los Millennials que alguna vez han comprado en línea, el $37 \%$ lo ha hecho desde un monedero electrónico (tarjetas de prepago), el $21 \%$ de una tarjeta de crédito o débito, en tercer lugar se encuentra Depósito bancario con $18 \%$, seguido del pago en efectivo (contra entrega) con el $16 \%$, y finalmente se encuentran las transferencias bancarias con el $8 \%$, la aplicación Paypal no es utilizada hasta el momento por los consumidores Millennials de comercio electrónico en Tulancingo, (Ver gráfica 12). 
ISSN: 1794-9920 Impreso / Electrónico 2500-9338

Enero - Julio de 2019 - Volumen 19 Número 2, Año 2019 Págs. 58 - 69

Gráfica 12: Forma de pago de las compras en línea.

¿QUÉ MÉTODO UTILIZA PARA PAGAR SUS COMPRAS EN LÍNEA?

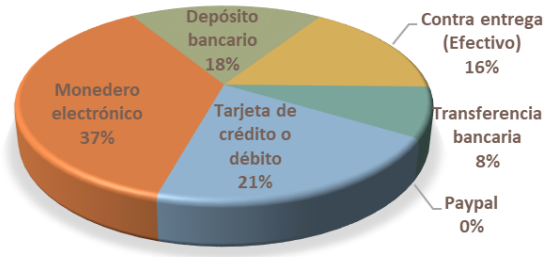

Fuente: Elaboración propia.

El tipo de productos que los Millennials adquieren por compras en línea, se encuentra $44 \%$ de los que han comprado Accesorios, ropa, zapatos; en segundo lugar, se encuentran los electrónicos con el $24 \%$, seguido de los productos de Hogar con el $13 \%$, un $10 \%$ ha comprado juguetes y $9 \%$ indica Otros, (Ver gráfica 13).

Gráfica 13: Tipos de productos en compras en línea.

¿QUÉ TIPOS DE PRODUCTOS HA COMPRADO EN LÍNEA?

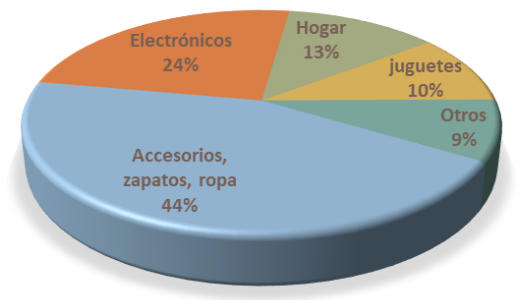

Fuente: Elaboración propia.

Con respecto a la pregunta de qué dispositivo es más usado para hacer la compra en línea, el smartphone es el preferido por los Millennials con un $84 \%$, seguido de la computadora con un $13 \%$, en tercer lugar, se encuentra las tabletas con el $3 \%$, mientras que otro no fue considerado por los encuestados con el $0 \%$, (Ver gráfica 14).

Gráfica 14: Compras online según dispositivo móvil más utilizado.

¿QUÉ DISPOSITIVO USA PARA HACER UNA COMPRA EN LÍNEA?

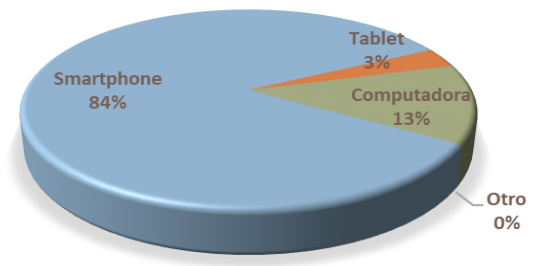

Fuente: Elaboración propia.
La percepción de los Millennials en cuanto a las compras en línea es que son seguras con el $71 \%$ y el $29 \%$ menciona que no son seguras, (Ver gráfica 15).

Gráfica 15: Percepción de seguridad en las compras en línea.

SEGURAS?

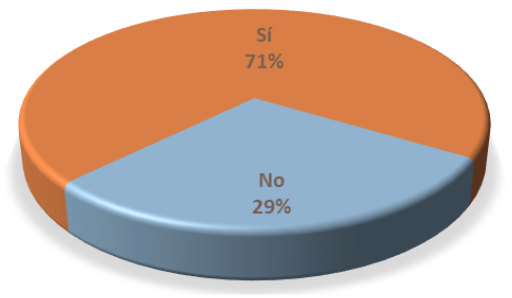

Fuente: Elaboración propia.

\section{REFERENCIAS:}

Berrocal, S. (2004). Una aproximación a la nueva retórica del líder político televisivo: acciones, cualidades y discurso. Doxa Comunicación 2, 53-67.

D'Adamo, O., García Beaudoux, V. y Pastore, M. (2015 Marzo). EL "PRESIDENTE IDEAL" PARA LOS PORTEÑ̃S. Centro de Opinión Pública.

Deusdad, B. (2003). El concepto de liderazgo político carismático: Populismo e identidades. Opción, 19(41), 9-35. Recuperado en 18 de febrero de 2019, de http://www.scielo.org.ve/scielo.php?script=sci_arttext\&p id=S1012-15872003000200002\&lng=es\&tlng=es.

Diemidova, A., Adamovska, V. y Garasiuk, O. (2018). Effective Image of Modern Politician in the Context of Public Management and Administration. Scientific Periodicals. 2, 41-49. 
García, V., D’Adamo, O. y Zubieta, E. (2016). Presidente ideal y Comunicación Política. Expectativas de los ciudadanos e imágenes proyectadas en los spots de las campañas presidenciales argentinas 2003, 2007 y 2011. Universidad Santiago de Compostela MARCO, 2, 1-23. http://dx.doi.org/10.15304/marco.2.2842

Gil Aluja, J. (2002). Introducción de la Teoría de la incertidumbre en la gestión de empresas. Vigo España: Editorial Milladoiro.

Kaufmann, A.; Gil Aluja, J. (1992). Técnicas de gestión de empresa: Previsiones, Decisiones y Estrategias. Madrid España: Ediciones Pirámide.

Kinder, D.R., Peters, M.D., Abelson, R.P. y Fiske, S.T. (1980). PRESIDENTIAL PROTOTYPES. Political Beahavior, 2(4), 315-337.

Martín Salgado, L. (2002). Marketing Político. Arte y ciencia de la persuasión en democracia. Barcelona España: Paidós Ibérica.

Merolla, J. y Zechmeister, E. (2009). Las percepciones de liderazgo en el contexto de las elecciones mexicanas de 2006. Política y Gobierno, Volumen Temático 1, 4181.

Ogneva Yu.I. y Kalmykov N.N. (2017). Expert Opinion: Image of the Ideal Politician in Russia. - Polis. Political Studies. 1 116-124. (In Russ.).DOl: https://doi.org/10.17976/jpps/2017.01.10

Orejuela S.S. (2009). Personalización política: la imagen del político como estrategia electoral. Revista de Comunicación, 8, 60-83.

Roets, A., y Van Hiel, A. (2009). The ideal politician: Impact of voters' ideology. Personality and Individual Differences, $\quad$ 46(1), 60-65. doi:10.1016/j.paid.2008.09.006

Zakrizevska, M. (2012). The social representations of the inhabitants of Latvia about the ideal image of a politician, EDP Sciences SHS Web of Conferences 2, 00042 DOI: 10.1051/shsconf/20120200042
Zamora, R. y Losada, J. (2011). La imagen del candidato electoral: Hacia una integración de su dimensión racional y emocional. Cuadernos de Información, 29, 924. 